\title{
Role of parents and peers in influencing the smoking status of high school students in Taiwan
}

\author{
C P Wen, S P Tsai, T Y Cheng, C C Hsu, T Chen, H S Lin
}

Tobacco Control 2005; 14(Suppl I):i10-i15. doi: 10.1136/tc.2003.005637

See end of article for authors' affiliations

......................

Correspondence to: Chi Pang Wen, National Health Research Institutes, 35 Keyan Road, Zhunan Town, Miaoli County, Taiwan 350; cwengood@ nhri.org.tw

\begin{abstract}
Objectives: To assess parental influence on smoking behaviour by high school students in an Asian culture and to compare the relative importance of parental and peer influence.

Methods: A 5\% nationally representative sample, including 44976 high school students in 10th to 12th grade (aged 15-18 years) in Taiwan, were surveyed in 1995. Each completed a long self administered questionnaire. Parental influence was measured by examining both parental behaviour (smoking status) and attitudes (perceived "tender loving care" (TLC) by adolescents). Changes in smoking status were used to determine peer influence, defined as the increase in the likelihood of smoking from grade 10 to 12 in a steady state environment. Odds ratios (ORs) were calculated for parental and peer influence, using logistic regression.

Results: Adolescents of smoking parents with low TLC had the highest smoking rates and those of nonsmoking parents with high TLC had the lowest. The difference was more than twofold in boys and more than fourfold in girls. When either parental smoking status or TLC alone was considered, parental influence was similar to peer influence in boys, but larger than peer influence in girls. However, when smoking status and TLC were considered jointly, it became larger than peer influence for both groups (OR 2.8 v 1.8 for boys and OR 3.9 v 1.3 for girls).

Conclusion: When parental influence is taken as parental behaviour and attitude together, it plays a more important role than peer influence in smoking among high school students in Taiwan. This study, characterising such relationships among Asian populations for the first time, implies that future prevention programmes should direct more efforts toward the parental smoking and parent-child relationships, and not aim exclusively at adolescents in schools.
\end{abstract}

c has long been recognised that adolescents do not try cigarettes in a vacuum, ${ }^{1}$ and "significant others", such as friends, classmates, siblings or parents, are the most consistent predictors of adolescent smoking. ${ }^{1-5}$ Among these "significant others", having smoking friend(s) has been considered as the most important factor influencing smoking of adolescents in the Western world, ${ }^{2-16}$ and such observation has been similarly recognised in Taiwan. ${ }^{17-21}$ Even though over half of the studies on youth smoking found parental influence playing a significant role, ${ }^{2}$ it has received limited attention and mixed reviews. ${ }^{82-26}$ Associating with peers who smoke is seen by researchers as an integral part of the initiation and progression process toward the development of smoking. ${ }^{127}$ Indeed, the efforts of most youth tobacco control programmes, including those in Taiwan, have been directed at school settings, partly because schools are easily accessed and partly because of the view that peer influence, not parental influence, is the most important determinant in smoking. ${ }^{28}$ Such a view was highlighted by the statement in the US Surgeon General's 1994 report that "parental influence was not as compelling as peer influence" ${ }^{29}$

Parental influence is culture specific but such influence on adolescent smoking in societies other than Western ones has rarely been examined. ${ }^{9}{ }^{10-32}$ Asian parenting style is known to be more authoritarian and expecting of more obedience. ${ }^{33}$ Even in Western societies, the justification for downplaying parental influence has been questioned. ${ }^{35}{ }^{36}$ In addition, this debate has yet to consider the importance of methodological differences in defining and quantifying "peer influence" and "parental influence" and these differences in definition have prevented a fair comparison. ${ }^{37}{ }^{38}$ Adolescent friendships are often formed or cemented on the basis of a common behaviour such as smoking, and, in these cases, "peer selection" rather than peer influence produces the association between friends and smoking. Several studies show that smoking adolescents actively seek out groups of friends with similar smoking behaviours and attitudes, ${ }^{39-41}$ in essence forming smoking cliques. ${ }^{13942-44}$ When friend selection, selected based on established smoking status, is counted as part of the peer influence, such influence will be overestimated..$^{52545}$ On the other hand, depending on how it is defined, parental influence is easily underestimated. Studies on parental influence are generally limited to the one common variable of parental smoking. Parental attitude, such as the level of caring support, or parental policies on smoking at home, may be as important as parental smoking, ${ }^{1}{ }^{46-48}$ and yet attitude has less often been considered as an independent factor when compared against peer influence. ${ }^{1}$ Unless attitude is considered together with behaviour, it appears likely that parental influence will be only partially assessed. ${ }^{38}{ }^{49}$ A recent review of 87 studies on familial influences on adolescent smoking concluded "methods are limited by a lack of standardized instruments, .... and the use of inconsistent definitions...". ${ }^{22}$ Because of these pitfalls in overestimating peer influence ${ }^{37}$ and underestimating parental influence, ${ }^{38}{ }^{49}$ it is important to re-examine the widely held belief that peer influence is the prime determinant, ${ }^{34}$ particularly in a population with an Asian culture.

Furthermore, peer influence has traditionally been studied by directly asking adolescents whether their commencement of smoking was influenced by peers. An answer here requires subjective retrospective recall of events and reaching a judgment on the determinants of a past behaviour. For

Abbreviations: $O R$, odds ratio; $R R$, rate ratio, $T L C$, tender loving care 
example, upon questioning by teachers, some students are likely to offer more "politically correct" or "expected" answers, and blame friends rather than parents. This pattern of responses is of particular concern, as these answers bias the results in only one direction and cannot be objectively validated. The use of direct questioning is also found to be problematic when adolescents are asked "whether their best friends smoke". ${ }^{522}$ An affirmative answer does not necessarily indicate a causal relationship, but more of a "rater effect", which tends to project their own behaviours and overestimate "friendship homophily". ${ }^{5}$ It may simply demonstrate that, because of common interests, smokers generally befriend other smokers. Given these methodological limitations, in this study we chose to use an alternative approach to assess peer influence. Peer influence was based, not on opinions, but on objective data-that is, the actual smoking status among the classmates or schoolmates. Using national survey data from Taiwan, this paper assesses parental influence on smoking among high school students in an Asian culture and compares the relative influence of peers and parents on smoking.

\section{METHOD}

In 1995, a nationwide survey of senior high school students (grades 10-12) was conducted to assess behavioural risk factors in Taiwan. A total of 44976 students were surveyed (girls 22821 , boys 22155 ). This was nearly 5\% of the national high school population of 959 081. Most students in the study were aged 15-18 years. The study represented students from all three high school types. In Taiwan, students enter high school through a testing system. Regular high schools have the highest academic standing and are considered the most prestigious, vocational high schools are the least prestigious, and five year junior colleges are in between. In the case of five year junior colleges, the first three years are equivalent to high school.

Each student completed an anonymous, self administered questionnaire on behavioural risk factors. A public health nurse collected the completed questionnaire and assured its confidentiality bypassing school administration. At the time of the survey, no parental consent was required and students were not informed that they could decline participation.

Each student completed a list of over 60 multiple choice questions, including two on smoking and eight on parentadolescent relationships. Smoking status questions were: (1) "Do you currently smoke?"-yes/no; and (2) "Is any member of your family a smoker?"-parents, siblings or others? (only answers of "parents" were used in this analysis). A correlation matrix compared answers from the eight parent-adolescent relationship questions (table 1 , footnote). All eight were found to be highly correlated (Cronbach's coefficient $\alpha$ 0.89). We therefore selected a single question ("On the whole, do you feel that your parents love you and care about you? i.e. How much TLC do you receive from your parents?"- -very high/high/medium/low/very low) to represent the adolescents' perception of parental level of tender loving care (TLC). This TLC answer showed significant correlation with each of the seven other answers $(r=0.43-$ 0.59). To facilitate statistical analysis the answers were grouped into two categories: high TLC (very high, high, and medium); and low TLC (low and very low).

Both univariate and multivariate logistic regression analyses were performed to calculate the rate ratios (RR) and odds ratios (OR), respectively, for parental influence and peer influence on adolescent smoking. Multivariate analysis included sex, type of school, peer influence, and parental influence as independent variables. Changes in smoking prevalence from the survey were used to determine peer influence, which was defined as the increase in the likelihood of smoking from grades 10 to 12 in a steady state environment. As discussed below, the assumption of a steady state in the external environment is made to exclude potential confounders affecting smoking. All statistical analyses were performed with SAS version 8.0.

\section{RESULTS}

Nearly a quarter $(24.5 \%)$ of boys smoked whereas smoking was approximately a fifth as prevalent $(5.1 \%)$ in girls (tables 1 and 2). Smoking rates generally increased stepwise through grades 10-12, in each type of high school, for both boys and girls. Regular high schools had the lowest smoking rates

Table 1 Smoking rates (\%) by parental behaviour (smoking) and attitude (TLC) †, by grade levels and by type of high school among boys

\begin{tabular}{|c|c|c|c|c|c|c|c|}
\hline & \multirow[b]{2}{*}{ Overall } & \multicolumn{2}{|c|}{ Parental smoking } & \multicolumn{2}{|l|}{ Parental TLC } & \multirow{2}{*}{$\begin{array}{l}\text { Non-smoking parents } \\
\text { with high TLC }\end{array}$} & \multirow{2}{*}{$\begin{array}{l}\text { Smoking parents } \\
\text { with low TLC }\end{array}$} \\
\hline & & No & Yes & High & Low & & \\
\hline Total & $24.5(22155)$ & 16.5 (8093) & $25.0 *(10810)$ & 20.3 (16617) & $29.3^{*}(5538)$ & $14.4(6305)$ & $31.0 *(2860)$ \\
\hline 10th grade & $18.6(7949)$ & 13.6 (2883) & $20.5^{*}(3966)$ & $15.9(5846)$ & $26.2^{*}(2103)$ & $11.2(2204)$ & $27.5^{*}(1093)$ \\
\hline 11 th grade & $22.3(7610)$ & $16.4(2774)$ & $24.8^{*}(3735)$ & $20.3(5717)$ & $28.3^{*}(1893)$ & $15.0(2166)$ & $30.9^{*}(1000)$ \\
\hline 12 th grade & $27.4(6596)$ & $20.1(2436)$ & $31.0^{*}(3109)$ & $25.2(5054)$ & $34.8^{*}(1542)$ & 17.5 (1935) & $36.1^{*}(767)$ \\
\hline Regular high school & $14.7(9350)$ & $10.7(3858)$ & $16.5^{*}(4212)$ & $12.7(7277)$ & $21.4^{*}(2073)$ & $8.9(3112)$ & $22.7^{*}(1002)$ \\
\hline 10th grade & $11.4(3085)$ & $8.4(1257)$ & $12.9^{*}(1401)$ & $9.7(2376)$ & $17.2^{*}(709)$ & 6.8 (991) & $19.2^{*}(329)$ \\
\hline 11 th grade & $14.4(3268)$ & $10.1(1341)$ & $16.2^{*}(1495)$ & $9.8(2546)$ & $20.9^{*}(722)$ & 8.8 (1094) & $22.1 *(371)$ \\
\hline 12 th grade & $18.3(2997)$ & $13.5(1260)$ & $20.8^{*}(1316)$ & $16.0(2355)$ & $26.6 *(642)$ & 11.1 (1027) & $27.2 *(302)$ \\
\hline \multicolumn{8}{|l|}{ Vocational high } \\
\hline school & 30.2 (9441) & $24.3(3004)$ & $32.2^{*}(4953)$ & $28.2(6688)$ & $35.1 *(2753)$ & $22.3(2200)$ & $36.4^{*}(1498)$ \\
\hline 10th grade & $25.8(3674)$ & 20.7 (1196) & $27.0 *(1955)$ & $22.7(2520)$ & $32.6^{*}(1154)$ & $18.0(867)$ & $32.9^{*}(641)$ \\
\hline 11 th grade & 30.1 (3204) & $24.3(980)$ & $32.1 *(1712)$ & $28.7(2286)$ & $33.6^{*}(918)$ & $23.6(705)$ & $36.1^{*}(502)$ \\
\hline 12 th grade & $36.8(2563)$ & $29.4(828)$ & $40.4^{*}(1286)$ & $35.0(1882)$ & $41.6^{*}(681)$ & $26.8(628)$ & $43.1 *(355)$ \\
\hline 5 year junior college & $22.6(3364)$ & $16.0(1231)$ & $24.9^{*}(1645)$ & $20.8(2652)$ & $29.5^{*}(712)$ & $14.3(993)$ & $31.7 *(360)$ \\
\hline 10th grade & $15.0(1190)$ & $9.1(430)$ & $17.1^{*}(610)$ & $13.4(950)$ & $21.7^{*}(240)$ & $6.9(346)$ & $21.1 *(123)$ \\
\hline 11 th grade & $22.9(1138)$ & $17.9(453)$ & $25.4^{*}(528)$ & $20.9(885)$ & $30.0 *(253)$ & $16.9(367)$ & $36.2 *(127)$ \\
\hline 12 th grade & $31.0(1036)$ & 22.1 (348) & $33.7 *(507)$ & $29.2(817)$ & $37.4^{*}(219)$ & $20.0(280)$ & $38.2^{*}(110)$ \\
\hline \multicolumn{8}{|c|}{$\begin{array}{l}\text { * } \mathrm{p}<0.05 \text {. } \\
\text { Numbers in parentheses indicate the number of students surveyed. } \\
\text { tTender, loving care (TLC) question on parental attitude perceived by students was selected from the following } 8 \text { questions: (1) On the whole, do you feel that your } \\
\text { parents love you and care about you? (2) On the whole, do you feel that your parents love you and understand you? (3) On the whole, do you feel that your } \\
\text { parents love you and trust you? (4) On the whole, do you feel that your parents love you and treat you fairly? (5) On the whole, do you feel that your parents love } \\
\text { you and respect you? (6) On the whole, do you feel happy about your family life? (7) On the whole, is the relationship good between you and your father? (8) On } \\
\text { the whole, is the relationship good between you and your mother? }\end{array}$} \\
\hline
\end{tabular}


Table 2 Smoking rates (\%) by parental behaviour (smoking) and attitude (TLC), by grade levels and by type of high school among girls

\begin{tabular}{|c|c|c|c|c|c|c|c|}
\hline & \multirow[b]{2}{*}{ Overall } & \multicolumn{2}{|c|}{ Parental smoking } & \multicolumn{2}{|l|}{ Parental TLC } & \multirow{2}{*}{$\begin{array}{l}\text { Non-smoking parents } \\
\text { with high TLC }\end{array}$} & \multirow{2}{*}{$\begin{array}{l}\text { Smoking parents } \\
\text { with low TLC }\end{array}$} \\
\hline & & No & Yes & High & Low & & \\
\hline Total & $5.1(22821)$ & $2.9(7694)$ & $6.0 *(11593)$ & $4.1(17281)$ & $8.2^{*}(5540)$ & $2.2(6074)$ & $9.2^{*}(3025)$ \\
\hline 10th grade & 4.9 (7511) & $2.7(2471)$ & $5.5^{*}(3896)$ & $3.8(5635)$ & $7.9^{*}(1876)$ & $2.0(1945)$ & $8.3^{*}(1063)$ \\
\hline $11^{\text {th }}$ grade & $4.8(7839)$ & $2.4(2651)$ & $5.8^{*}(3999)$ & $3.8(5860)$ & $7.8^{*}(1979)$ & $1.6(2074)$ & $8.8^{*}(1076)$ \\
\hline 12th grade & $5.6(7471)$ & 3.5 (2572) & $6.7^{*}(3698)$ & $4.6(5786)$ & $9.0 *(1685)$ & $3.0(2055)$ & $10.6^{*}(886)$ \\
\hline Regular high school & $2.8(9165)$ & $1.5(3535)$ & $3.5^{*}(4306)$ & $2.3(7278)$ & $4.8^{*}(1887)$ & $1.0(2907)$ & $5.0^{*}(990)$ \\
\hline 10th grade & $2.6(3013)$ & $1.2(1156)$ & $3.3^{*}(1414)$ & $2.0(2364)$ & $4.6^{*}(649)$ & $0.7(955)$ & $4.8^{*}(357)$ \\
\hline 11 th grade & $2.8(3162)$ & $1.4(1186)$ & $3.4^{*}(1521)$ & $2.1(2458)$ & $5.1 *(704)$ & $0.6(949)$ & $5.5^{*}(362)$ \\
\hline 12th grade & $3.0(2990)$ & $1.9(1193)$ & $3.7^{*}(1371)$ & $2.7(2456)$ & $4.5^{*}(534)$ & $1.6(1003)$ & $4.4^{*}(271)$ \\
\hline Vocational high school & 7.5 (10723) & $4.7(3112)$ & $8.4^{*}(5854)$ & $6.1(7646)$ & $10.9^{*}(3077)$ & $3.9(2317)$ & $12.1^{*}(1755)$ \\
\hline 10th grade & $7.6(3.540)$ & $4.9(993)$ & $7.9^{*}(1999)$ & 6.4 (2487) & $10.3^{*}(1053)$ & $4.3(720)$ & $11.0 *(611)$ \\
\hline $11^{\text {th }}$ grade & $6.8(3571)$ & $3.8(1039)$ & $7.9^{*}(1959)$ & $5.4(2525)$ & $10.3^{*}(1046)$ & 2.8 (788) & $11.2^{*}(614)$ \\
\hline 12th grade & $8.0(3612)$ & $5.3(1080)$ & $9.3^{*}(1896)$ & $6.4(2634)$ & $12.3^{*}(978)$ & $4.6(809)$ & $14.5^{*}(530)$ \\
\hline 5 year junior college & $3.6(2933)$ & $2.3(1047)$ & $4.1 *(1433)$ & $3.2(2357)$ & $4.9(576)$ & $1.9(850)$ & $5.4^{*}(280)$ \\
\hline 10th grade & $2.0(958)$ & $1.2(322)$ & $2.1(483)$ & $1.2(784)$ & $5.8^{*}(174)$ & $0.4(270)$ & $4.2^{*}(95)$ \\
\hline $11^{\text {th }}$ grade & $4.1(1106)$ & $2.1(426)$ & $5.0 *(519)$ & 4.0 (877) & $4.4(229)$ & $1.8(337)$ & $6.0 *(100)$ \\
\hline 12th grade & $4.6(869)$ & $3.7(299)$ & $5.1(431)$ & $4.6(696)$ & 4.6 (173) & 3.7 (243) & $5.9(85)$ \\
\hline
\end{tabular}

(overall boys $14.7 \%$, girls $2.8 \%$ ), and vocational schools the highest (overall boys $30.2 \%$, girls $7.5 \%$ ) with five year junior colleges intermediate (boys $22.6 \%$, girls $3.6 \%$ ). These smoking rates from the three types of high schools differed significantly $(\mathrm{p}<0.01)$, for both boys and girls.

Among boys, one quarter $(25 \%)$ with smoking parents smoked, in contrast to $16.5 \%$ with non-smoking parents (table 1). The smoking rate among those reporting low TLC was $29.3 \%$, compared to $20.3 \%$ for those with high TLC. In the presence of both low TLC and smoking parents, $31.0 \%$ of boys smoked as opposed to $14.4 \%$ with non-smoking and high TLC parents, or a difference of over 2:1. Parental smoking rate was $57.2 \%$ among male students (not shown).

Among girls, $6 \%$ with smoking parents smoked, in contrast to $2.9 \%$ with non-smoking parents (table 2 ). The smoking rate among those reporting low TLC was $8.2 \%$, compared to $4.1 \%$ of those with high TLC. In the presence of both low TLC and smoking parents, $9.2 \%$ smoked, as opposed to $2.2 \%$ with high TLC and non-smoking parents, or a difference of over
4:1. Parental smoking rate was $60.1 \%$ among female students (not shown).

If parents smoked, their children had a higher chance of smoking ( 1.7 times for boys and 2.2 times for girls) than if parents did not smoke (table 3). Similar results were seen if students received low TLC (RR 1.6 for boys and 2.1 for girls). Students with smoking and low TLC parents were 2.7 times more likely to smoke than students with non-smoking and high TLC parents in boys and 4.4 times in girls. The above analysis was repeated for students in each of the three different types of schools. Results were virtually the same as those shown in table 3.

The increase in the likelihood of smoking from 10th to 12th grade was assessed among students with parents of different smoking status and different TLC (table 3 ). The RRs ranged between 1.5 and 1.9 for boys and 1.1 and 1.5 for girls. Nearly all RRs were significant.

Additional analysis using a multivariate model, adjusting for sex and type of school, was also conducted to compare

\begin{tabular}{|c|c|c|}
\hline Influencing factors & Boys & Girls \\
\hline \multicolumn{3}{|l|}{ Parental factors } \\
\hline \multicolumn{3}{|l|}{ A. Smoking } \\
\hline Non-smoking parent & 1.0 & 1.0 \\
\hline Smoking parent & $1.7(1.6$ to 1.8$)$ & $2.2(1.8$ to 2.5$)$ \\
\hline \multicolumn{3}{|l|}{ B. Tender loving care (TLC) } \\
\hline High TLC parent & & \\
\hline Low TLC parent & $1.6(1.5$ to 1.7$)$ & $2.1(1.9$ to 2.4$)$ \\
\hline \multicolumn{3}{|l|}{ C. Smoking and low TLC co-existed } \\
\hline Non-smoking with high TLC parent & 1.0 & 1.0 \\
\hline Smoking with low TLC parent & $2.7(2.4$ to 3.0$)$ & $4.4(3.6$ to 5.5$)$ \\
\hline \multicolumn{3}{|c|}{ Peer factor } \\
\hline \multicolumn{3}{|c|}{ A. Student with non-smoking and high TLC parent } \\
\hline 10th grade & & \\
\hline 12th grade & $1.7(1.4$ to 2.0$)$ & $1.5(1.0$ to 2.3$)$ \\
\hline \multicolumn{3}{|c|}{ B. Student with non-smoking and low TLC parent } \\
\hline 10th grade & & 1.0 \\
\hline 12th grade & $1.6(1.2$ to 2.1$)$ & $1.1(0.6$ to 1.8$)$ \\
\hline \multicolumn{3}{|c|}{ C. Student with smoking and high TLC parent } \\
\hline 10 th grade & & 1.0 \\
\hline 12th grade & $1.9(1.7$ to 2.2$)$ & $1.2(1.0$ to 1.6$)$ \\
\hline \multicolumn{3}{|c|}{ D. Student with smoking and low TLC parent } \\
\hline 10th grade & 1.0 & 1.0 \\
\hline 12th grade & 1.5 (1.2 to 1.8$)$ & $1.3(1.0$ to 1.8$)$ \\
\hline
\end{tabular}


Table 4 Odds ratios (OR) for factors affecting smoking behaviours of high school students using multivariate logistic regression

\begin{tabular}{|c|c|c|c|}
\hline Influencing factors & Boys & Girls & Total \\
\hline Peer influence ${ }^{*}$ & 1.8 (1.6 to 2.0$)$ & $1.3(1.1$ to 1.5$)$ & $1.7(1.5$ to 1.8$)$ \\
\hline \multicolumn{4}{|l|}{ Parental influence $\dagger$} \\
\hline Smoking & $1.8(1.6$ to 2.0$)$ & $2.0(1.6$ to 2.5$)$ & $1.8(1.6$ to 2.0$)$ \\
\hline Low TLC & $2.1(1.8$ to 2.5$)$ & $2.2(1.6$ to 3.1$)$ & $2.1(1.8$ to 2.4$)$ \\
\hline Combined effect (smoking and low TLC) & $2.8(2.4$ to 3.2$)$ & $3.9(3.0$ to 5.0$)$ & $3.0(2.7$ to 3.4$)$ \\
\hline
\end{tabular}

parental influence and peer influence on student smoking (table 4). Overall, the peer influence was similar to parental smoking factor (OR $1.7 v 1.8$ ), but was weaker than parental TLC factor (OR $1.7 v 2.1)$. For girls, either one of the parental factors showed greater influence over the peer factor (OR 2.0 and $2.2 v 1.3$ ). When parental influence was jointly assessed from both factors, smoking and low TLC, parental influence became substantially larger than peer influence (OR $2.8 v 1.8$ for boys and OR $3.9 \vee 1.3$ for girls). Additional findings showed that parental TLC was not remarkably different from parental smoking status, although OR for TLC (2.1) was slightly larger (1.8).

\section{DISCUSSION}

Although both peer influence and parental influence played an important role in determining the adolescents' smoking, this study shows that parental influence, as a whole, was more important than peer influence in this Asian population. Either parental factor taken alone, smoking or low TLC, exerted more influence on girls' smoking than did their peers. With boys, the combination of the two parental factors had more influence than peers. These observations, based on a nationally representative sample, are at variance with the prevailing opinion in the Western literature, which holds that peer influence is more important than parental influence. ${ }^{2522} 2627$ In Asian culture, the relatively authoritarian parenting style and prevailing submissive adolescent mindset are important factors in influencing adolescents, compared to their counterparts in Western culture. The high rate of smoking $(46 \%)$ and low rate of cessation $(7 \%)^{50}$ among male adults in Taiwan, reflective of parental behaviour and attitudes toward smoking, would also exert more of the negative parental influence than in a society where smoking rate is low and cessation rate is high.

This study shows the importance of how peer or parental influence is defined in leading to the conclusion on their relative strength. Determining whether peer influences actually cause others to use tobacco is notoriously difficult. ${ }^{1}$ In addition, it is likely to confuse peer influence with peer selection. Friends are selected at the beginning of the relationship, while influence is developed over the course of the friendship. ${ }^{5}$ Peer influence is traditionally studied by directly asking students whether their smoking was influenced by peers. Such direct questioning on the source of his or her smoking not only leads to results that are subjective and difficult to verify but, more seriously, tends to overestimate peer influence. ${ }^{51}$ Overestimation arises in two scenarios. First, researchers interpret peer relationship as being responsible for initiating or perpetuating smoking while, in fact, friends have often selected each other on the basis of already established common interests (for example, smoking). ${ }^{52}$ Some smokers seek out other smokers for places to smoke or for conversation during smoking, while others befriend those smokers sharing common characteristics such as adventurousness, rebellious attraction, or supportive of smoking behaviour. ${ }^{1}$ Consistent with the adage that "birds of a feather flock together", research suggests that teenagers belong to friendship groups with those who are similar to themselves. ${ }^{5}$ In reviewing five studies attempting to separate selection from influence effects, selection has been concluded to be as important if not more important than influence. ${ }^{42}{ }^{51}{ }^{53-55}$ Second, there is a tendency among study adolescents to give "expected" or "politically correct" answers. It has been reported as a form of "projection" as adolescents tend to attribute their own behaviours to friends. ${ }^{37}$

In this study, an alternative approach has been taken. Instead of asking for individual judgment on why they smoke, we used changes in the level of smoking prevalence among classmates or schoolmates as an assumed average influence that a student is exposed to. The more your classmates smoke the more affected you will be. While our study is concerned more with average group smoking behaviour, it could be argued that the intensity of peer influence differs with each individual and the use of group data may have masked such variability.

Most studies assume that the critical determinant of parental influence is whether they smoke or not. ${ }^{22}$ This influence is obvious when youngsters hold smoking parents as a role model to imitate. ${ }^{256}$ In addition, having cigarettes easily available in the home certainly facilitates adolescent experimentation. $^{257}$ However, we have found that parental influence is much more than simply their smoking behaviour. Parental attitude, which may be expressed in terms of tolerance toward smoking or the extent of TLC exhibited toward children, is another important dimension of parental influence. Ours is one of very few studies comparing the dual parental factors of smoking behaviour and perceived parental attitude. $^{8275859}$ Two studies that did take this approach found that the joint effect was much more powerful than individual effect. Nolte et al reported a 10-fold synergistic relationship in youth smoking between smoking parents who were permissive and non-smoking parents who disapproved of smoking. ${ }^{49}$ Newman replicated this study and found a smaller, though still significant, threefold difference. ${ }^{38}$ In contrast, a smaller relationship was found in both studies (twofold or less), when only one variable (behaviour) was considered. In our study, parental smoking yielded odds ratios of 1.8 for boys and 2.0 for girls. However, when attitude (TLC) was added, the relationship became much larger, 2.8 for boys and 3.9 for girls. These results confirm the notion that parental influence has been underestimated in studies when only one aspect of parenting was considered.

The need for a steady state assumption is important and is a prerequisite for comparing peer influence exerted over a period of time. In reviewing 87 studies on peer and familial influences, "failure to measure important confounding and mediating factors" has been noted. ${ }^{22}$ Other than peers and parents, external events could affect the smoking status of the adolescents during this period of time. They include 
activities having either positive or negative impact on smoking status. ${ }^{2}$ In both cross sectional and longitudinal study designs, such a steady state assumption is needed to hold external confounders constant in order to make a valid comparison of peer influence over time. When the study period is relatively short and few external events had affected smoking, as in this study, this steady state assumption is not unreasonable.

We applied several epidemiological principles of causality to assess the validity of the findings - that is, strength, consistency, and temporal relationship. The strength of the relationship is strong and significant. Results are consistent, not only for boys and for girls, but also consistently observed for each type of school in each sex. That girls were more influenced by parents than boys in this study, is consistent with Asian cultural norm where girls are more obedient than boys in accepting parental advice. ${ }^{60}$ Several other studies have also reported, as in this study, that parental influence was equal $^{245861-64}$ or more important than peer influence. ${ }^{65-67}$ When smoking rates of adolescents and adults were compared in 24 states in the USA, they were found to be highly correlated-that is, states with higher adult smoking rates showed higher adolescent smoking rates, supporting the aetiological importance of the parental role. ${ }^{68}$ As to temporal relationship, parents usually have intimate relationships, much earlier than peers, by starting from early infancy. All children want to imitate parents from early childhood. ${ }^{11}{ }^{69-72}$ Parents generally have much longer and more frequent contact with their children than any peer claiming to have influence over them. Most parents usually remain throughout one's adolescence, while peers or best friends change frequently. ${ }^{13}$ For example, over a two year period in a longitudinal study, more than half of the high school smokers and non-smokers changed peer groups. ${ }^{39}$

Several tobacco control advocates (Glantz, ${ }^{36} \mathrm{Hill}^{74}$ Males $^{35}{ }^{68}$ ) have raised strong arguments for emphasising the reduction of adult smoking as the mainstay to reduce youth smoking. This is because "School based programs attacking peer influence could be counterproductive by making smoking alluring as a forbidden, adults-only habit" ${ }^{1}{ }^{75}$ Such a view is fully consistent with the implications arising from this study that parental influence plays a critical role in their youth smoking.

These findings have important implications for Asian countries. School programmes should include more efforts on the parental factors, and not exclusively directed at adolescents on campus. ${ }^{67} 75$ Parents could become motivated by the awareness of the negative role they play in their children's lives and the potential of dual benefits if they quit smoking. ${ }^{76}{ }^{77}$ As noted by Jacobson et al in the book Combating teen smoking, interventions that inform parents about the message that their smoking behaviour or tolerance toward smoking sent to children should be emphasised. ${ }^{1}$

There are a number of limitations to this study. First, the measure of smoking is based on a single question about whether the respondent currently smokes, but "current smokers" in this survey did not follow the conventional definition. To assess the potential impact from differences in definition, we compared the prevalence of smokers in this study with that of a more recent national survey which used the World Health Organization definition of "current smokers". ${ }^{50}$ The results were virtually the same. For example, the smoking prevalence of current smokers among boys in the recent survey were $19.3 \%, 22.6 \%$, and $27.5 \%$ for grade 10 , 11 , and 12 , respectively, ${ }^{78}$ as compared to $18.6 \%, 22.3 \%$, and $27.4 \%$ from this study. Second, this study used "perceived level of tender loving care" from parents as an indicator of the parental relationship, and did not use the commonly used parenting style (such as authoritative). However, as this

\section{What this paper adds}

In Taiwan, peer influence, traditionally viewed as the critical factor in affecting adolescent smoking, is not as important as parental influence, when both parental smoking and parental attitudes are considered together. Previous studies have overestimated peer influence and underestimated parental influence because they were defined differently. Youth smoking intervention programmes should focus more on parental smoking and parent-child relationships, and not aim exclusively at adolescents in schools. The role of adults, both their behaviours and attitudes, is too important to be ignored in any youth prevention programme.

question was found to be highly correlated with seven other questions in the same domain, it does reflect the parenting style of the adolescents. Third, data were presented on parental smoking without separating fathers from mothers. This is because our study was focused more on parental smoking as whole, and the number of smoking mothers was too small to provide a reliable estimate. (The smoking rate for fathers was $56 \%$, while the rate for mothers $5 \%{ }^{78}$ ) Fourth, peer influence was defined as any factor other than parental influence, and this may have led to an overestimation of the peer influence. Given that the study is to assess the relative strength between the two, our study result on parental influence would, therefore, be on the conservative side. Fifth, the measures used to assess the parental influence were not comprehensive. For example, parental smoking intensity was not available and could not be analysed. Finally, the difference in smoking rates was based on a cross sectional survey rather than a longitudinal follow up. Given the large sample size, short time lapse and the steady state assumption, the impact of the difference in study design would be negligible.

Despite these limitations, we believe this study, based on nationally representative data, demonstrates that parental influence plays as important or more important role than peer influence in affecting adolescent smoking.

\section{Authors' affiliations}

C P Wen, T Y Cheng, C C Hsu, Division of Health Policy Research, National Health Research Institutes, Miaoli, Taiwan

S P Tsai, The University of Texas Health Science Center at Houston, School of Public Health, Houston, Texas, USA

T Chen, Tulane University School of Public Health, New Orleans, Louisiana, USA

H S Lin, Department of Public Health, Chung Shan Medical University, Taichung, Taiwan

Competing interests: none declared

\section{REFERENCES}

1 Jacobson $\mathrm{P}$, Lantz $\mathrm{P}, \mathrm{W}$ arner $\mathrm{K}$, et al. Combating teen smoking: research and policy strategies. Ann Arbor, Michigan: University of Michigan Press, 2001.

2 Tyas SL, Pederson LL. Psychosocial factors related to adolescent smoking: a critical review of the literature. Tobacco Control 1998;7:409-20.

3 Institute of Medicine. Growing up tobacco free: preventing nicotine addiction in children and youth. Washington DC: Institute of Medline, National Academy Press, 1994.

4 US Department of Health and Human Services. Reducing tobacco use: a report of the Surgeon General. Atlanta, Georgia: US Department of Health and Human Services, Centers for Disease Control and Prevention, National Center for Chronic Disease Prevention and Health Promotion, Office on Smoking and Health, 2000

5 Kobus K. Peers and adolescent smoking. Addiction 2003;98:s37-55

6 Biglan A, Duncan TE, Ary DV, et al. Peer and parental influences on adolescent tobacco use. J Behav Med 1995;18:315-30.

7 Moore L, Roberts C, Tudor-Smith C. School smoking policies and smoking prevalence among adolescents: multilevel analysis of cross-sectional data from Wales. Tobacco Control 2001;10:117-23. 
8 Hu FB, Flay BR, Hedeker D, et al. The influences of friends' and parental smoking on adolescent smoking behavior: the effects of time and prior smoking. J Applied Soc Psychology 1995;25:2018-47.

9 Shibata A, Fukuda K. Hirohata T. Smoking habits among senior high school students and related factors. Kurume Med J 1990;37:129-40.

10 Ogawa H, Tominaga S, Gellert G, et al. Smoking among junior high school students in Nagoya, Japan. Int J Epidemiol 1988;17:814-20.

11 Wang S, Yu J, Zhu B, et al. Cigarette smoking and its risk factors among senior high school students in Beijing, China, 1988. Tobacco Control 1994;3:107-14.

12 Conrad KM, Flay BR, Hill D. Why children start smoking cigarettes: predictors of onset. Br J Addict 1992:87:1711-24.

13 Pasvola M, Vartiainen E, Puska P. Predicting adult smoking: the influence of smoking during adolescence and smoking among friends and family. Health Educ Res 1996;11:309-15.

14 Stanton WR, Lowe JB, Silva PA. Antecedents of vulnerability and resilience to smoking among adolescents. J Adolesc Health 1995:16:71-7.

15 Poulsen LH, Osler M, Roberts $C$, et al. Exposure to teachers smoking and adolescent smoking behaviour: analysis of cross sectional data from Denmark. Tobacco Control 2002;11:246-51.

16 Zhu B, Liu $M$, Shelton $D$, et al. Cigarette smoking and its risk factors among elementary school students in Beijing. Am J Public Health 1996:86:368-75.

17 Chen HS, Percy MS, Horner SD. Cigarettes: a growing problem for Taiwanese adolescents. J Community Health Nurs 2001; 18:167-75.

18 Chou P, Liou MY, Lai MY, et al. Time trend of substance use among adolescent students in Taiwan, 1991-1996. J Formos Med Assoc 1999:98:827-31.

19 Yang MS, Yang MJ, Liu YH, et al. Prevalence and related risk factors of licit and illicit substances use by adolescent students in southern Taiwan. Public Health 1998;112:347-52

20 Wang CS, Chou P. The prevalence and motivating factors of adolescent smoking at a rural middle school in Taiwan. Subst Use Misuse 1996:31:1447-58.

21 Chen KT, Chen CJ, Fagot-Campagna A, et al. Tobacco, betel quid, alcohol, and illicit drug use among 13- to 35-year-olds in I-Lan, rural Taiwan: prevalence and risk factors. Am J Public Health 2001;91:1130-4.

22 Avenevoli S, Merikangas KR. Familial influences on adolescent smoking. Addiction 2003;98:1-20.

23 Distefan JM, Elizabeth BA, Gilpin A, et al. Parental influences predict adolescent smoking in the United States, 1989-1993. J Adolesc Health 1998:22:466-74.

24 Flay BR, Hu FB, Richardson J. Psychosocial predictors of different stages of cigarefte smoking among high school students. Prev Med 1998;27:A9-18.

25 Urberg KA, Degirmencioglu SM, Pilgrim C. Close friend and group influence on adolescent cigarette smoking and alcohol use. Dev Psychol 1997:33:834-44.

26 Bauman KE, Carver K, Gleiter K. Trends in parent and friend influence during adolescent: the case of adolescent cigarette smoking. Addict Behar 2001; 26:349-61

27 Flay BR, Hu FB, Siddiqui O, et al. Differential influence of parental smoking and friends' smoking on adolescent initiation and escalation of smoking. $J$ Health Soc Behar 1994;35:248-65.

28 Centers for Disease Control and Prevention. Guidelines for school health programs to prevent tobacco use and addiction. MMWR Morb Mortal Wkly Rep 1994:43:1-18.

29 US Department of Health and Human Services. Preventing tobacco use among young people. A report of the Surgeon General, 1994. Atlanta, Georgia: Public Health Service, Centers for Disease Control and Prevention, Office on Smoking and Health, 1994 (US Government Printing Office Publication No S/N 017-001-00491-0.).

30 Lee A, Tsang CK. Youth risk behaviour in a Chinese population: a territorywide youth risk behavioural surveillance in Hong Kong. Public Health 2004:118:88-95.

31 Hesketh T, Ding QJ, Tomkins A. Smoking among youths in China. Am J Public Health $2001 ; 91: 1653-5$.

32 Jayant K, Notani PN, Gulati SS, et al. Tobacco usage in school children in Bombay, India. A study of knowledge, attitude and practise. Indian I Cancer 1991:28:139-47.

33 Lin CC, Fu VR. A comparison of child-rearing practice among Chinese, immigrant Chinese, and Caucasian-American parents. Child Dev 1990;61:429-33.

34 Steinberg L, Dornbusch S, Brown BB. Ethnic differences in adolescent achievement: an ecological perspective. Am Psychologist 1992;47:723-9.

35 Males $\boldsymbol{M}$. The influence of parental smoking on youth smoking: Is the recent downplaying justified? I School Health 1995;65:228-31.

36 Glantz S. Preventing tobacco use - the youth access trap. Am J Public Health 1996:86:1156-8.

37 Bauman KE, Ennett ST. On the importance of peer influence for adolescent drug use: commonly neglected considerations. Addiction 1996;91:185-98.

38 Newman IM, Ward JM. The influence of parental attitude and behavior on early adolescent cigareet smoking. J School Health 1989;59:150-2.

39 Engels RC, Knibbe RA, Drop MJ, et al. Homogeneity of cigarette smoking within peer groups: influence or selection? Health Educ Behav 1997;24:801-11.

40 Thrush D, Fife-Schaw C, Breakwell GM. Young people's representations of others' view of smoking: Is there a link with smoking behaviour? J Adolesc 1997;20:57-70.

41 Fergusson DM, Lynskey MT, Horwood $\amalg$. The role of peer affiliations, social family and individual factors in continuities in cigarette smoking between childhood and adolescence. Addiction 1995:90:647-59.

42 Ennett ST, Bauman KE. The contribution of influence and selection to adolescent peer group homogeneity: the case of adolescent cigarette smoking $J$ Pers Soc Psychol 1994;85:589-93.
43 Urberg KA. Locus of peer influence: social crowd or best friend. J Youth Adolesc 1992;26:763-78.

44 Pederson LL, Koval JJ, McGrady GA, et al. The degree and type of relationship between psychosocial variables and smoking status for students in grade 8: is there a dose-response relationship? Prev Med 1998;27:337-47.

45 Bauman KE, Ennett ST. Peer influence in adolescent drug use. Am Psychologist 1994;49:820-2.

46 Reimers TM, Pomrehn P, Becker SL, et al. Risk factors for adolescent cigarette smoking. Am J Dis Child 1990:144:1265-73.

47 Jackson C, Dickinson D. Can parents who smoke socialise their children against smoking? Results from the smoke-free kids intervention trial. Tobacco Control 2003;12:52-9.

48 Farkas AJ, Gilpin EA, White MM, et al. Association between household and workplace smoking restrictions and adolescent smoking. JAMA 2000;284:717-22.

49 Nolte AE, Smith BJ, O'Rourke T. The relative importance of parental attitudes and behavior upon youth smoking behavior. I School Health 1983:53:264-71.

50 Wen CP, Levy DT, Cheng TY, et al. Smoking behaviour in Taiwan, 2001. Tobacco Control 2005; 14(suppl I): i51-5.

51 Kandel DB, Demise B. The parental and peer contexts of adolescent deviance: an algebra of interpersonal influences. J Drug Issues 1996;26:289-316.

52 Kandel DB. Homophily, selection and socialization in adolescent friendship. Am J Sociol 1978;84:427-37.

53 Aesltine RH. A consideration of parental and peer influences on adolescent deviance. J Health Soc Behav 1995;36:103-21.

54 Cohen JM. Source of peer group homogeneity. Sociol Educ 1977;30:241-77.

55 Billy JO, Udry JR. Patterns of adolescent friendship and effects on sexual behavior. Soc Psychol Q 1985;48:27-41.

56 Geckova A, van Dijk JP, van lttersum-Gritter T, et al. Determinants of adolescents' smoking behaviour: a literature review. Cent Eur J Public Health 2002;10:79-87.

57 Woodruff SI, Candelaria JI, Laniado-Laborin R, et al. Availability of cigarettes as a risk factor for trial smoking in adolescents. Am J Health Behav 2003;27:84-8

58 Gottlieb NH. The effects of peer and parental smoking and age on the smoking careers of college women: a sex-related phenomenon. Soc Sci Med 1982:16:595-600

59 Biglan A, Duncan TE, Ary DV, et al. Peer and parental influences on adolescent tobacco use. J Behav Med 1995;18:315-30.

60 Gershoff ET. Corporal punishment by parents and associated child behaviors and experiences: a meta-analytic and theoretical review. Psychol Bull 2002;128:539-79.

61 O'Loughlin J, Paradis G, Renaud L, et al. One-year predictors of smoking initiation and of continued smoking among elementary schoolchildren in multiethnic, low-income, inner-city neighbourhoods. Tobacco Control 1998; 7:268-75.

62 Zhang L, Wang W, Zhao Q, et al. Psychosocial predictors of smoking among secondary school students in Henan, China. Health Educ Res 2000;15:415-22.

63 Dusenbury L, Kerner JF, Baker E, et al. Predictors of smoking prevalence among New York Latino youth. Am J Public Health 1992;82:55-8.

64 Greenlund KJ, Johnson CC, Webber LS, et al. Cigarette smoking attitudes and first use among third- through sixth-grade students: the Bogalusa heart study. Am J Public Health 1997;87:1345-8.

65 Jarallah JS, Bamgboye EA, Al-Ansary LA, et al. Predictors of smoking among male junior secondary school students in Riyadh, Saudi Arabia. Tobacco Control 1996;5:26-9

66 Molyneux A, Lewis A, Antoniak M, et al. Is smoking a communicable disease? Effect of exposure to ever smokers in school tutor groups on the risk of incident smoking in the first year of secondary school. Tobacco Control 2002; 11:241-5.

67 Bauman KE, Foshee VA, Linzer MA, et al. Effect of parental smoking classification on the association between parental and adolescent smoking. Addict Behav 1990;15:413-22.

68 Males M. Smoked: why Joe Camel is still smiling. Common Courage Press. 1999.

69 Stanton WR, Silva PA. Children's exposure to smoking. Int I Epidemiol 1991;20:933-7.

70 Lau RR, Quadrel MJ, Hartman KA. Development and change of young adults' preventive health beliefs and behavior: Influence from parents and peers. J Health Soc Behav 1990;31:240-59.

71 Brook U, Mendelberg A, Galili A, et al. Knowledge and attitudes of children towards cigarette smoking and its damage. Patient Educ Couns 1999:37:46-53.

72 Andrews EL, Hearne JJ. Effects of primary grades health curriculum project on student and parent smoking attitudes and behavior. I School Health 1984;54:18-20.

73 Krosnick JA, Judd CM. Transition in social influence at adolescence: who induces cigarette smoking? Dev Psychol 1982;18:359-68.

74 Hill D. Why we should tackle adult smoking first. Tobacco Control 1999;8:333-5

75 Flay BR. Psychological approaches to smoking prevention: a review of findings. Health Psychol 1985:4:449-88.

76 Guest Editorial Opinion. Pro and con: should smoking cessation be given more emphasis than smoking prevention? Family Practice News 2000.

77 Swan AV, Creeser R, Murray M. When and why children first start to smoke. Int J Epidemiol 1990;19:323-31.

78 National Health Research Institutes. National Health Interview Survey (NHIS) in 2001. Taiwan: National Health Research Institutes, 2002, http:// nhis.nhri.org.tw. 